\title{
Review of: "Uremic serum damages endothelium by provoking excessive neutrophil extracellular trap formation"
}

\author{
Laureline Berthelot ${ }^{1}$ \\ 1 French Institute of Health and Medical Research
}

Potential competing interests: The author(s) declared that no potential competing interests exist.

In this manuscript, the authors showed that endothelial cells exposed to NET from neutrophil derived HL-60 cells induced by serum from CKD patients exhibited increased apoptosis. Moreover, CKD patients with poor outcome have higher levels of markers of NETosis. I have some points to be addressed:

- How the authors selected the $40 \mathrm{HD}$ patients for the in vitro study? The description only mentions HD patients. Do they belong to the group with poor or good outcome? Did the authors compare these two distinct group in vitro? The sera were selected because of their high level of NET? Uremic toxin?

- Can the authors add information on CKD patients? They mentioned that most of patients were diabetic, but what about patients with inflammatory kidney diseases? These kinds of disease could also affect the production of NETs like ANCA disease.

- In the statistic description, Anova with t test were performed. But in the cellular study, only 20 patients composed the groups. Did the author check for the normal distribution of the data?

- Minor point: page 3, in the "differentiation of HL-60 cells" part, the abbreviations DMSO, ATRA and FACS were not detailed. 